\title{
ENFERMAGEM EM DOENÇAS TRANSMISSÍVEIS: COMO ABORDAR ESSE TEMA NA GRADUAÇÃO EM ENFERMAGEM?
}

\section{NURSING IN TRANSMISSIBLE DISEASES: HOW TO DISCUSS THIS TOPIC IN NURSING GRADUATION?}

\author{
Lislaine Aparecida Fracolli* \\ Lúcia Yasuko Izumi Nichiata** \\ Renata Ferreira Takahashi* \\ Maria Amélia de Campos Oliveira* \\ Anna Luiza de F.P.L. Gryschek**
}

FRACOLLI, L.A. et al. Enfermagem em doenças transmissiveis: como abordar esse tema na graduação em enfermagem? Rev.Esc.Enf.USP, v. 34, n.4, p. 395-400, dez. 2000.

\section{RESUMO}

Este trabalho relata o esforço da Disciplina de Enfermagem em Saúde Coletiva coin enfoque nas Doenças Transmissiveis (ESC-DT) para reorganizar seu objeto de ensino, seu o conteúdo teórico-prático e suas estratégias pedagógicas, tendo como finalidade contribuir para a formação de um profissional de enfermagem comprometido com a Saúde Coletiva. Para tanto, busca-se descrever o contexto em que se inscreve a Saúde Coletiva, bem como o perfil do profissional de saúde que se faz necessário para a implementação do Projeto Sistema Único de Saúde. Relata-se o conteúdo e as estratégias de desenvolvimento da experiência de ensino e faz-se uma análise crítica das potencialidades e limites da mesma para a obtenção da finalidade proposta.

PALAVRAS-CHAVE: Enfermagem. Ensino-aprendizagem. Doenças transmissiveis. Saúde coletiva.

\section{ABSTRACT}

This study relates the attempts of the discipline Collective Health Nursing with focus in transmissible diseases to reorganize its teaching object, its theorical and practical content, and its pedagogical atrategics, as a contribution to the formation of a nursing professional comnpromissed with Collective Health. It descrybes the context of Collective Health in Brazil and the characteristics needed to improve the implementation of the Unified Health Care System in the Country. It also reports the content and the padagogical strategics used in the discipline and makes a critical analysis of its potentialities and limitations to the porposed goal

KEYWORDS: Nursing. Teaching-learning. Transmissible diseases. Colective health.

\section{O CONTEXTO HISTÓRICO DA SAÚDE COLETIVA}

Nesta segunda metade do século XX, a humanidade experimenta rápidas e profundas transformações em todas as esferas da vida econômica, cultural, social e política.

A globalização da economia tem como conseqüências a transnacionalização empresarial, adesterritorialização da força de trabalho e o desemprego estrutural, entre outras. Estas conseqüências coexistem com o aumento das desigualdades entre os povos e os grupos sociais, a eclosão de movimentos nacionalistas, a exacerbação dos conflitos étnicos, a agressão ao meio ambiente, a

Enfermeira. Professora Doutora do Departamento de Enfermagem em Saúde Coletiva da Escola de Enfermagem da Universidade de São Paulo (EEUSP). E-mail: lislaine@usp.br

* $\quad$ Enfermeira, Professora Assistente do Departamento de Enfermagem em Saúde Coletiva da Escola de Enfermagem da Universidaáe de São Paulo, Doutoranda da EEUSP . 
deterioração do espaço urbano, a intensificação da violência e o desrespeito aos direitos humanos. (PAIM; ALMEIDA FILHO, 1998)

$\mathrm{Na}$ saúde, ampliou-se o debate sobre as relações entre o desenvolvimento econômico e o social que marcaram a década de 60 para, a partir dos anos 70, sustentar uma discussão sobre a extensão da cobertura dos serviços públicos de saúde. $O$ reconhecimento do direito à saúde e a responsabilidade da sociedade em garantir os cuidados básicos de saúde, estabelecido a partir da Conferência de Alma-Ata, foram questionados pelos grandes centros hegemônicos da economia mundial. Estes revalorizam o mercado como o mecanismo privilegiado para a alocação de recursos e questionam a responsabilidade estatal na provisão de bens e serviços para o atendimento das necessidades sociais, inclusive a saúde.

Nos anos oitenta, a ordem mundial que se instaurou inspirada no neoliberalismo provocou uma marcante fragilização dos esforços públicos para o enfrentamento coletivo dos problemas de saúde. Particularmente nos países de economia capitalista dependente, a opção pelo "Estado Mínimo" e o corte de gastos públicos comprometeram em muito o campo social conhecido como Saúde Pública. (PAIM; ALMEIDA FILHO, 1998)

Esse contexto inaugurou um período de "crise" da Saúde Pública, percebida de modo diferente pelos distintos sujeitos que atuam nesse campo social. Para a superação dessa crise, diferentes aportes sociais têm sido propostos, cada um deles indicando novos paradigmas para a "Saúde Pública". Segundo PAIM; ALMEIDA FILHO (1998), dentre os diferentes sujeitos sociais que têm formulado propostas para a superação da crise da Saúde Pública estão os defensores da Saúde Coletiva.

Para EGRY (1996), a Saúde Coletiva vem se configurando como campo de saber e prática de saúde em nosso país desde a década de 70 e caracteriza-se por entender o processo saúde-doença como social e historicamente determinado, por incorporar o método materialista-dialético e as ciências sociais no estudo dos fenômenos da saúde e por se preocupar com a Saúde Pública como a saúde do público, sejam indivíduos, grupos étnicos, gerações, castas, classes sociais ou populações.

AYRES (1997) considera que toda situação de crise constitui-se em uma possibilidade favorável para a revisão de valores, conceitos e práticas. Assim, frente à "crise" do setor público de saúde no Brasil, este autor aproveita para questionar alguns aspectos fundamentais que tem direcionado o pensar/agir em Saúde Pública como, por exemplo: onde está o público hoje? como é construído? quem são e onde estão seus sujeitos? o que os motiva e move no cotidiano? que relações guardam os diversos agravos à saúde com essas motivações e movimentos? como se busca hoje compreender e transformar os processos coletivos de adoecimento?

Esse mesmo autor afirma ainda que aplicar em larga escala os avanços da tecnologia biomédica é tão pouco potente para resolver os problemas de saúde na sua dimensão sanitária, quanto insistir que só com mudanças sociais profundas conseguir-se-á transformar a realidade de saúde no Brasil.

AYRES (1997) aposta que a potência para essa transformação encontra-se na estimulação dos movimentos microssociais como forma de tecer e sustentar mudanças efetivas na saúde da população. Considera ainda que a tarefa da Saúde Pública neste final de século deveria ser identificar, compreender e ressocializar as motivações e os movimentos microssociais dos sujeitos, configurados mediante a experiência compartilhada e cotidiana da saúde e do adoecimento.

Diante dessa concepção e das dificuldades para superar o baixo impacto das tradicionais intervenções da Saúde Pública, PAIM; ALMEIDA FILHO (1998) questionam qual deve ser o perfil do profissional de saúde para atuar nesse contexto em transformação. Que conteúdos devem ser privilegiados na sua formação? Se o campo de conhecimento da Saúde Pública é tão amplo que não comporta um tipo único de profissional para atuar nas instituições de saúde, qual o núcleo básico de conhecimentos e habilidades que devem compor o novo perfil profissional. Tendo-se em vista um contexto de saúde que incorpora a cada dia novos atores para o seu âmbito de ação, exigindo cada vez mais capacidade de negociação, agir comunicativo e administração de conhecimentos, quais são os requisitos para conduzir um trabalho cada vez mais coletivizado?

Segundo RODRIGUES (apud PAIM; ALMEIDA FILHO, 1998), o avanço em direção a uma prática de saúde semelhante à acima descrita necessita de profissionais que sejam capazes de cumprir distintas atribuições. Estas variam desde uma função histórico-politica de "resgatar, do próprio processo histórico de construção social da saúde, os conhecimentos, êxitos e fracassos da humanidade em sua luta pela cidadania e bem estar"; até uma função agregadora de valor, através da produção e da gestão do conhecimento científico-tecnológico. Em outras palavras, profissionais que possam assumir desde a função estratégica de gerência de recursos escassos, de mediador estratégico das relações entre as necessidades e os problemas de saúde e as decisões políticas, até uma função de "advocacy", utilizando o conhecimento como um instrumento de denúncia, para promover a mobilização crescente da sociedade em direção à realização do seu potencial de saúde e pelo exercício do direito de cidadania.

As instituições acadêmicas e de serviços de saúde não podem ignorar o movimento que se engendra em 
torno da formulação de uma política global de saúde. Ao contrário, devem buscar dialogar e fazer alianças com as instituições governamentais e não governamentais para o enfrentamento dos problemas de saúde, aproveitando seus espaços de atuação para produzir novos conceitos, teorias e práticas que, num processo de "interfertilização", poderão influir nas políticas e práticas de saúde, numa dimensão mais geral. (PAIM; ALMEIDA FILHO, 1998).

Nesse sentido, a idéia é que se desenvolvam processos de formação profissional que se caracterizem por serem sistemáticos, criativos e inovadores e cujos eixos fundamentais sejam a investigação e a articulação com os serviços de saúde.

Para PAIM; ALMEIDA FILHO (1998), o marco conceitual que tem orientado o ensino, a pesquisa e a extensão em Saúde Coletiva, no caso brasileiro, baseia-se nos seguintes pressupostos :

- a saúde como estado vital (setor de produção e campo do saber), está articulada à estrutura da sociedade através das suas instâncias econômica e politico-ideológica, possuindo portanto historicidade;

- as ações de saúde (promoção, proteção, recuperação, reabilitação) constituem práticas sociais e trazem consigo as influências das relações entre os grupos sociais;

- o objeto da Saúde Coletiva é construído nos limites do biológico e do social e compreende a investigação dos determinantes da produção social das doenças e da organização dos serviços de saúde, bem como o estudo da historicidade do saber e das práticas sobre os mesmos. Nesse sentido, o caráter interdisciplinar desse objeto sugere uma integração no plano do conhecimento e não somente no plano da estratégia de reunir profissionais com múltiplas formações ;

- o conhecimento não se dá pelo contato com a realidade, mas pela compreensão das suas leis e pelo comprometimento com as forças capazes de transformá-la.

O desenvolvimento desse núcleo conceitual pressupõe a realização de estudos relativos:

- ao fenômeno saúde/doença como processo social;

- à investigação da produção e da distribuição das doenças na sociedade, como resultado de processos de produção e de reprodução social;

- à analise das práticas de saúde (processos de trabalho), na sua articulação com as demais práticas sociais;

- à compreensão das formas como a sociedade identifica e explica suas necessidades e problemas de saúde e organiza-se para enfrentá-los.
Dessa maneira, o ensino de saúde desenvolvido dentro do campo de conhecimento da Saúde Coletiva deve buscar superar o biologicismo dominante, a naturalização da vida social, a submissão à clinica e a dependência do modelo médico-hegemônico (PAIM; ALMEIDA FILHO, 1998).

\section{O CASO DA DISCIPLINA DE ENFERMAGEM EM SAÚDE COLETIVA COM ENFOQUE NAS DOENÇAS TRANSMISSÍVEIS (ESC-DT) E SUA INSCRIÇÃO NO CAMPO DA SAÚDE COLETIVA}

Segundo TAKAHASHI e cols (1997), as doenças infecciosas situam-se entre os temas mais complexos e diversificados da clínica e da epidemiologia, além de conformarem um panorama epidemiológico cuja disseminação configura a sua persistente associação com as condições de vida das populações.

Para essas autoras, a expansão para outros segmentos sociais das epidemias, antes restritas aos bolsões de pobreza, pôs em cheque as formas de interpretação e intervenção a elas dirigidas. As diretrizes ministeriais recomendando a abolição das unidades específicas de isolamento e a exigência de que as normas de biossegurança fossem incorporadas no cotidiano do trabalho em saúde nos diferentes níveis de assistência e em quaisquer instituições para toda a clientela também tiveram uma expressiva parcela explicativa na mudança radical dos paradigmas de ensino/pesquisa/intervenção que direcionam o trabalho voltado às Doenças Transmissiveis (DT) .

Segundo TAKAHASHI e cols (1997), as ações de prevenção, controle e tratamento das DT competem ao conjunto dos trabalhadores da saúde, que contribuem cada qual com seus conhecimentos ehabilidades específicos para a finalidade do Processo de Produção de Serviços de Saúde. As intervenções junto aos indivíduos, grupos ou comunidades acometidos por DT têm como finalidade última a transformação dos perfis epidemiológicos da população no que tange à essas doenças.

A enfermagem, ao integrar o processo de trabalho em saúde para assistir aos pacientes portadores de DT, desenvolve intervenções individualizadas, ao atender às alterações biopsicossociais desencadeadas pelo processo saúdedoença na dimensão singular'. Desenvolve ainda intervenções de monitoramento do processo infeccioso e dos perfis referentes aos processos de adoecer e morrer dos grupos e comunidades, intervenções centradas mais especificamente nas dimensões particular e geral do processo saúdedoença, articuladas à Vigilância Epidemiológica.

\footnotetext{
${ }^{1}$ A dimensão singular diz respeito aos individuos e suas famílias, a dimensão particular aos grupos sociais e a dimensão estrutural, aos processos mais gerais de estruturação da sociedade. (EGRY, 1996)
} 
As tentativas de viabilizar o SUS no Brasil, a adesão à proposta de intervir sobre os determinantes e condicionantes do processo saúde-doença (e não apenas sobre os seus resultados), a adoção da Vigilância à Saúde como eixo do trabalho em saúde, entre outros, favoreceram a inscrição da disciplina de Enfermagem em Doenças Transmissíveis no campo da Saúde Coletiva, o que em significa, em última instância, abordar a questão das DT numa dimensão mais particular, enfatizando as tecnologias relativas à Vigilância Epidemiológica.

Segundo o Centro de Vigilância Epidemiológica do Estado de São Paulo (BRASIL/CVE,1998), Vigilância Epidemiológica (VE) define-se como o conjunto de atividades que permitem reunir a informação indispensável para conhecer a qualquer momento o comportamento ou história natural das doenças, bem como detectar ou prever alterações de seus fatores condicionantes, com o fim de recomendar oportunamente sobre bases firmes as medidas indicadas e eficientes que levem à prevenção e ao controle de determinadas doenças. Esse grupo considera também que são funções da VE a coleta de dados, o processamento dos dados coletados, a análise e a interpretação dos dados processados, a recomendação das medidas de controle apropriadas, a promoção das ações de controle indicadas, a avaliação da eficácia e efetividade das medidas adotadas e a divulgação de informações pertinentes.

TAKAHASHI e cols (1997), ao definir a atuação da enfermagem junto à VE, consideram que a mesma desenvolve ações de Investigação Epidemiológica, de diagnóstico situacional, de planejamento e de implementação de medidas de prevenção, controle e tratamento das DT. Apontam ainda como tecnologias necessárias para a enfermagem no desempenho dessas ações a visita domiciliar, a habilidade de comunicação e o relacionamento interpessoal com indivíduos, grupos e comunidades.

Contudo, "formar" enfermeiros(as) capazes de articular na sua prática profissional, os diversos saberes e agires relativos ao controle das DT na sua dimensão mais particular, com uma visão "coletiva" do processo saúde-doença, num contexto de mudanças paradigmáticas e de crise dos serviços de saúde, vem se constituindo em um desafio para a Disciplina de ESC-DT. O enfrentamento desse desafio tem levado a disciplina a reconstruir sua trajetória de ensino e a realizar revisões sistemáticas de seu conteúdo teórico, de sua estratégia pedagógica e dos campos utilizados para o seu ensino prático.

Este trabalho pretende compartilhar a experiência vivenciada pelas docentes da disciplina ESC-DT, com outros profissionais que atuam na formação de recursos humanos em saúde e que sentem de perto as mudanças causadas pela crise do setor saúde e por isso buscam reconstruir suaspráticas de ensino e de intervenção no que tange à Saúde Coletiva e às doenças transmissiveis .

\section{A ESTRUTURA BÁSICA DA DISPLINA ESC- DT DA ESCOLA DE ENFERMAGEM DA UNIVERSIDADE DE SÃO PAULO (EEUSP)}

A disciplina de ESC-DT, desenvolvida junto ao Depto de Saúde Coletiva da Escola de Enfermagem da Universidade de São Paulo (EEUSP-SP), possui uma carga horária de 165 horas, distribuídas em períodos de 4 horas durante 9 semanas consecutivas. Seu público é constituído por discentes dos $2^{\circ}$ e $3^{\circ}$ anos do curso de graduação em enfermagem, o que caracteriza uma clientela heterogênea, totalizando cerca de 40 alunos por turma.

O propósito dessa disciplina é permitir que os estudantes expressem sua compreensão e reflitam sobre os problemas de saúde da população, buscando articular os mesmos com as forma de viver e trabalhar dos individuos/grupos, desenvolvendo para tanto habilidades de observação, leitura, interrelação e comunicação com indivíduos/grupos. Devem utilizar principalmente os saberes advindos da epidemiologia, das ciências sociais, da informática e da biologia.

As estratégias pedagógicas utilizadas baseiamse nos principios da metodologia da problematização, que considera que o objeto do conhecimento faz parte do real e tem que ser "descoberto", "apreendido" de forma ativa pelo aluno, sendo que o professor é um facilitador desse processo de descoberta do real.

Para propiciar as experiências pedagógicas capazes de levar o aluno a apreender a realidade das DT nos coletivos, foram desenvolvidas as seguintes atividades:

1- visita a UBS, com o objetivo de conhecer a organização do serviço de saúde, os problemas de saúde existentes naquela localidade, os registros de morbidade e mortalidade existentes, a população que freqüenta o serviço de saúde e as demandas por saúde que ela apresenta. A síntese dessa atividade foi realizada em uma aula expositiva, com indicação de textos para leitura, que teve como tema central o SUS e a determinação social do processo saúdedoença, objetivando que os alunos visualizassem a articulação entre os modos de vida das pessoas e os potenciais de saúde/doença das mesmas.

2 - leitura e discussão pelos alunos, de textos explicativos sobre cadeia de transmississão das Doenças Transmissiveis identificadas nos registros de morbidade do serviço de saúde visitado. A síntese dessa atividade foi realizada em uma reunião plenária em que foram discutidas a cadeia de transmissão das doenças e as formas de intervenção para interrompê-las. 
3- leitura e discussão dos Programas Estaduais para o controle da TUBERCULOSE, HANSENÍASE e DST/Aids e visita a Unidades de Saúde que desenvolviam tais programas. $\mathrm{O}$ objetivo dessa atividade foi discutir com os alunos as formas que o poder público tem adotado para intervir em algumas doenças transmissiveis de maior impacto social e o trabalho da enfermagem junto desses programas .

4 - desenvolvimento por parte dos alunos de práticas de Vigilância Epidemiológica relacionadas à analise de dados e planejamento de intervenções. A atividade foi organizada da seguinte maneira : a partir do Programa de DST/Aids, disponibilizou-se para os alunos o banco de dados sobre a epidemia de Aids no município de São Paulo, que a divisão de Epidemiologia do Programa Municipal de Aids tem estruturado. Este banco de dados contém informações da ficha de notificação compulsória de Aids, relativas aos casos de Aids notificados no município. A tarefa dos alunos foi organizar esses dados e de forma a descrever a evolução da epidemia e identificar a relação desses dados com as formas de vida e trabalho das pessoas (ou seja a determinação social do processo saúde/doença frente à Aids). Deveriam também descrever como se encontravam distribuídos os casos de Aids nos diferentes distritos do município de São Paulo. Como parte dessa tarefa, foram instrumentalizados em informática para o manejo do EPI-INFO, um software que possibilita a análise mais concisa e rápida dos dados. A síntese dessa atividade foi a apresentação de um relatório descritivo/explicativo da epidemia de Aids no município, relatório esse que foi apresentado e discutido com técnicos do setor de VE do Programa de DST/Aids. O objetivo dessa atividade pedagógica foi permitir aos alunos exercitar ações de Vigilância Epidemiológica comumente exercidas por enfermeiros em Unidades de Saúde.

5 - vivenciar atividades teórico-práticas da Investigação Epidemiológica e do Programa de Imunizações em Unidades Básicas de Saúde.

Buscou-se com isso que os alunos apreendessem a realidade de um serviço de saúde e formulassem, a partir das diferentes experiências vivenciadas, uma concepção própria a respeito da questão das DT nos serviços de saúde.

As habilidades técnicas exploradas durante o processo de ensino/aprendizagem foram principalmente a consulta de enfermagem, a visita domiciliária, a técnica de administração de medicamentos, a construção de gráficos e tabelas e a organização de apresentações públicas. As tecnologias envolvidas nessas habilidades, usandose aqui a nomenclatura de MERHY(1997), constituiram-se em tecnologias predominantemente do tipo leve (tecnologias de relações) e leve-dura (saberes, equipamentos).

A avaliação desse processo de ensino/ aprendizagem congregou três situações diferenciadas: a análise do desempenho dos alunos durante as atividades de campo, a apresentação do relatório descritivo sobre a questão da epidemia de Aids no municipio de São Paulo e uma prova escrita que buscou identificar a incorporação dos conceitos de VE e Determinação social do Processo Saúde-Doença na apreciação crítica dos Programas de Saúde analisados.

\section{POSSIBILIDADES E LIMITES DESSA PROPOSTA DE ENSINO}

Existem duas grandes lógicas que encaminham as inovações estruturais da disciplina de DT: o processo pedagógico e o foco sobre o objeto de intervenção. No que tange à questão pedagógica, pôde-se observar uma alteração na relação professor/ aluno/conhecimento, em que o aluno deixa de ser um sujeito passivo e passa a adotar uma postura mais ativa no processo de ensino/aprendizagem. O objeto do processo de ensino/aprendizagem não é "dado", aparece como algo multidimensional que está encoberto e que precisa ser conhecido. Olhados sob essa ótica, os limites da disciplina aparecem na pequena carga horária que a mesma apresenta, pois a tarefa de construção do conhecimento tem tempos variados para os diferentes sujeitos do processo de ensino-aprendizagem. Tal fato acarreta uma incompatibilidade entre o volume deosconteúdos a serem ministrados e as atividades propostas, frente ao tempo que o aluno tem para desenvolvê-las.

Outras questões encontradas foram a dificuldade dos alunos em aceitar esse novo papel no .processo de ensino/aprendizagem, a falta de estrutura fisica da EEUSP-SP para albergar processos de ensino que envolvam a utilização da informática ou a articulação entre diferentes campos de conhecimento, numa proposta de ensino integrado.

Com relação ao objeto de intervenção da enfermagem enfocado na disciplina de ESC-DT, um limite no desenvolvimento da proposta pedagógica foi a dificuldade de encontrar espaços de ensino prático que não estivessem presos ao modelo médico hegemônico, de cunho biologicista. Quando esses locais eram encontrados, existiam dificuldades de acesso aos mesmos, em função da distância geográfica ou do espaço físico inapropriado para receber o numero de alunos demandados pela disciplina. Dessa forma, a apreensão do conceito de determinação social do processo saúde/doença deuse parcialmente e principalmente como uma reflexão teórica, do que propriamente como uma "experiência" concretamente vivenciada pelos alunos, o que certamente prejudicou a apreensão do objeto de ensino, no caso as doenças transmissiveis, sob essa ótica. Ao avaliar a disciplina, os alunos relatam que as atividades 
práticas são as que mais impactam para seu processo de ensino/aprendizagem.

Um outro limite da experiência de ensino desenvolvida foi a dificuldade, por parte dos docentes, de equacionarem com segurança os contornos (em termos de quantidade e qualidade) da disciplina no conjunto das que integram o Departamento de Enfermagem em Saúde Coletiva e no currículo do curso de graduação da EEUSP-SP.

As possibilidades dessa experiência residem na oportunidade de organização de um processo de ensino/ aprendizagem que obtém como produto alunos mais críticos, criativos e capazes de conhecer e identificar algumas expressões das determinações do processo de trabalho em saúde e a especificidade da enfermagem no conjunto dos trabalhadores de saúde, podendo assim tornarem-se agentes ativos das transformações sociais.

A mudança de enfoque no objeto de ensino da disciplina de DT, privilegiando a estratégia da Vigilância Epidemiológica e não especificamente a fisiopatologia das DT, oportuniza a criação de um espaço potencial para a construção de um olhar menos "biológico", menos dependente da clínica, mais centrado nos coletivos e, consequentemente, com maior potencial para a superação do biologicismo e da submissão à clínica que tradicionalmente tem marcado a prática de enfermagem .

Tomar as DT dentro do trabalho de VE como eixo de ensino da disciplina de ESC-DT, pressupõe uma revisão das estratégia de intervenção de enfermagem em saúde coletiva e aponta para a necessidade de construção de novas tecnologias analíticas, interpretativas e interventivas que, incorporadas à VE, possam colaborar significativamente para a construção de saberes e técnicas no campo da Saúde Coletiva.

\section{ALGUMAS CONSIDERAÇÕES FINAIS}

A aproximação da Disciplina de ESC-DT aos conceitos de Vigilância a Saúde, de Determinação Social do Processo Saúde-Doença e de metodologia problematizadora, vem ocorrendo como parte de um processo que se articula à capacitação docente, à estruturação da realidade dos serviços de saúde e à politica de educação no Brasil.

Considerando que a realidade estrutura-se fora do âmbito de intervenção do conhecimento científico, existe a necessidade de uma constante revisão destes eixos epistemológicos, buscando garantir que os mesmos sempre possibilitem uma apreensão práxica do real. Assim, as alterações construídas na disciplina de Enfermagem em Saúde Coletiva com Enfoque nas Doenças Transmissiveis sinalizam a pertinência e atualidade dos eixos escolhidos pela mesma.

A diminuição de atividades práticas em campos hospitalares não se constitui em um fator que prejudica a formação do aluno no curso de graduação emenfermagem, no que tange às DT, visto que os conteúdos relativos à assistência de enfermagem em hospitais são amplamente trabalhado durante os anos de graduação da EEUSP. Além disso, em termos de biossegurança, não existe mais a conceituação da Unidade de Isolamento como o único local capaz de receber com segurança pacientes portadores de DT. Hoje, com as precauções padrão e as precauções baseadas nas formas de transmissão, vê-se que qualquer serviço e qualquer profissional de saúde pode (e deve) apropriar-se e aplicar os conhecimentos de biossegurança na sua prática assistencial.

A disciplina de Enfermagem em Saúde Coletiva com Enfoque nas DT entende que cada vez mais deve buscar conteúdos teóricos e práticos que the possibilitem construir saberes para intervir no processo saúde-doença das DT nos coletivos, dada a necessidade de construção de intervenções eficazes, sendo que o campo das intervenções individuais e voltadas ao corpo biológico encontra-se bem mais organizado.

No âmbito da Saúde Coletiva, vem-se discutindo a VE como uma ação que incorpora o registro e acompanhamento não só das doenças de notificação compulsória, mas dos agravos de saúde que acometem os sujeitos, ou seja as doenças crônico-degenerativas, o tabagismo, entre outros. Enfatiza-se ainda, a necessidade de uma descentralização e regionalização da VE, convocando os municipios a participar mais efetivamente dessas ações no seu território geo-social, sugerindo aos mesmos a assunção do planejamento e a implementação de ações de saúde consensuadas com as diversas instâncias governamentais que atuam na VE.

\section{REFERÊNCIAS BIBLIOGRÁFICAS}

ARANTES, C.I.S. Saúde Coletiva: os (des)caminhos da construção do ensino de enfermagem, São Paulo, 1999. 200 p. Tese (Doutorado) - Escola de Enfermagem, Universidade de São Paulo.

AYRES, J.R.C.M. Sobre o risco: para compreender a epidemilogia. São Paulo, HUCITEC, 1997.

BRASIL. Ministério da Saúde. Centro de Vigilância Epidemiológica. Guia de Vigilância Epidemiológica. Brasília, 1998

EGRY, E.Y. Saúde Coletiva: construindo um novo método em enfermagem. São Paulo, Ícone, 1996.

PAIM, J.; ALMEIDA FILHO, N. Saúde coletiva: uma "nova saúde pública" ou um campo aberto a novos paradigmas? Rev. Saúde Públ., v.32, n.4, p. 299-316, 1998.

MERHY, E.E. Em busca do tempo perdido: a micropolitica do trabalho vivo em saúde. In: MERHY, E.E.; ONOCKO, R.(org)

Agir em saúde: um desafio para o público. São Paulo. HUCITEC, 1997.

TAKAHASHI, R.F. et al. Intervenções de enfermagem em infectologia. In: VERONESI, R.; FOCACCIA, R. Tratado de Infectologia. São Paulo, ATHENEU, 1997. Cap 126, p. 1535 